\title{
Synthesis of novel 2-substituted 1,4-naphthoquinones using Heck reaction in 'green' reaction media
}

\author{
Alka Mital ${ }^{\mathrm{a} *}$, Rahul Lad ${ }^{\mathrm{a}}$, Ashish Thakur ${ }^{\mathrm{a}}$, Villendra Singh Negi ${ }^{\mathrm{a}}$ \\ and Uma Ramachandran ${ }^{b}$ \\ ${ }^{a}$ Department of Pharmaceutical Technology, National Institute of Pharmaceutical Education and \\ Research(NIPER), Sector-67, S.A.S. Nagar,Mohali-160062, India \\ ${ }^{b}$ Orchid Chemicals \& Pharmaceuticals Ltd., 476/14, Old Mahabalipuram Road \\ Sholinganallur, Chennai-600 119, India \\ E-mail: alkamital@gmail.com
}

\begin{abstract}
1,4-Naphthoquinones have been known to possess a wide spectrum of biological activities such as antidiabetic, antibacterial, antimalarial, anticancer and antifungal activities. An efficient, environmentally friendly two step process for synthesizing various 2-substituted 1,4naphthoquinones is described, starting from 2-bromonaphthalene and olefinic substrates using the Heck reaction in an ionic liquid (IL) followed by oxidation. It provides for a rapid and "green" methodology for the synthesis of the biologically active 2-substituted 1,4naphthoquinones.
\end{abstract}

Keywords: 1,4-Naphthoquinones, Heck reaction, ionic liquids, green chemistry, ligand-free palladium catalyst

\section{Introduction}

Quinones are useful compounds as synthetic intermediates and biologically active compounds and the 1,4-naphthoquinone structure constitutes an essential part of these classes of compounds. These are associated with antidiabetic, anticancer, antimalarial, antibacterial, antiinflammatory and antifungal activities ${ }^{1-4}$. Various 2-substituted 1,4-naphthoquinone derivatives can be prepared with ease starting from 2-bromonaphthalene using the Heck coupling reaction ${ }^{5}$, followed by oxidation ${ }^{6}$. Herein, we report the synthesis of new 2-substituted 1,4naphthoquinones for evaluating their biological activities.

More and more chemical and pharmaceutical industries are opting for replacing environmentally hazardous organic solvents in various chemical processes with environmentally friendly alternatives like ILs as green media ${ }^{7}$. The palladium catalyzed carbon-carbon bond forming Heck reaction can be successfully carried out in various ILs ${ }^{8,9}$. Seddon et al. first 
reported the Heck reaction of aryl halides and aromatic aldehydes with alkenes using homogeneous palladium complex catalysts and phosphine ligands in 1-butyl-3methylimidazolium hexafluorophosphate $\left([\mathrm{bmim}] \mathrm{PF}_{6}\right){ }^{10}$. The Heck reaction has also been reported to be carried out in imidazolium salts based ILs in phosphine ligand free systems ${ }^{11,12}$, if catalytic amount of halide is used in the reaction mixture in the form of tetrabutylammonium bromide (TBAB) or tetrabutylammonium chloride (TBACl).

Though 2-substituted naphthalenes can be prepared, using the Knoevenagel condensation reaction between naphthalene 2-carboxaldehyde and the corresponding reactive methylene compounds, we choose to obtain the same using the Heck reaction as it is known to give highly trans stereospecificity.

Moreover, there are only few reports ${ }^{13}$ of Heck reaction on naphthalenes and we proposed to study the effect of ILs on the rate and selectivity of these reactions. We have efficiently carried out the Heck coupling of 2-bromonaphthalene with various olefinic esters including $\alpha$ substituted olefins in the ionic liquids, TBAB and [bmim] $\mathrm{PF}_{6}$. The hydrophobic IL could be separated from the products and salt by-products by extraction through simple decantation of organic solvent and water, respectively. The yields obtained while using [bmim] $\mathrm{PF}_{6}$ and $\mathrm{TBAB}$ as the solvent for the reactions were greater than those obtained with conventional solvents like DMF. The products obtained in these reactions were predominantly $\mathrm{E}$ isomers. The IL containing palladium catalyst could be recycled several times without any loss in activity. The Heck coupling products were oxidized in a single step reaction in acetonitrile using chromium trioxide and periodic acid as the terminal oxidant to obtain the corresponding 2-substituted 1,4naphthoquinones in 50-60 \% yields.

Moreover, comparable yields and product selectivity to reactions in which ligand was used in the IL medium were obtained, if these reactions were carried out in the presence of catalytic amount of TBAB along with the palladium catalyst without any phosphine ligand. The 2substituted 1,4-naphthoquinones have been submitted for the screening of various biological activities and the results will be published soon.

\section{Results and Discussion}

In our efforts to standardize a suitable green reaction media for the synthesis of a wide range of 2-substituted 1,4-naphthoquinones, we used Heck coupling in ILs as the first step. Thus, substrate like 2-bromonaphthalene was subjected to Heck coupling with various olefinic esters. When 2, 4, and 5 were reacted with 2-bromonaphthalene in different ILs such as [bmim] $\mathrm{PF}_{6}$ and TBAB using palladium acetate as a catalyst with or without phosphine ligand and triethylamine as the base (Scheme 1), the results obtained are summarized in Table 1.

The Heck reactions were successfully carried out in [bmim] $\mathrm{PF}_{6}$ using palladium acetate and tris-o-tolylphosphine as ligand at $70{ }^{\circ} \mathrm{C}$ to give consistently better yields (Entries 2, 6, 8, 10, 13, 15, 18 in Table 1) compared to that done at $100{ }^{\circ} \mathrm{C}$ (Entry 3). The reactions carried out in 
TBAB as the IL gave low yields as compared to that done in [bmim] $\mathrm{PF}_{6}$ by a range of $10-15 \%$. This is expected, because the intermediate catalytic species involved in the Heck coupling in alkylimidazolium ILs are the Pd (0) dicarbene stabilized complexes generated in situ ${ }^{14}$.

Further, in order to study the halide effect on the Heck reaction in [bmim] $\mathrm{PF}_{6}$, catalytic amount of $\mathrm{TBAB}$ was added in [bmim] $\mathrm{PF}_{6}$ under previously optimized conditions without the use of phosphine ligand. The yields were quite comparable with those observed in [bmim] $\mathrm{PF}_{6}$ alone and the reaction was completed in a shorter time (Entries $\mathbf{4 , 7 , 9 , 1 1 , 1 4 , 1 6 , 1 9 ) . ~ T h e ~ I L ~}$ and the catalyst could be recycled several times without any decrease in reaction yields.

The oxidation of the 2-substituted naphthalenes was carried out in acetonitrile using chromium trioxide and periodic acid as the terminal oxidant to obtain corresponding naphthoquinones in 50-60 \% yields.

In conclusion we have developed an efficient method for the synthesis of 2-substituted 1,4naphthoquinones in simple two steps. The first step involves the Heck reaction in ILs like TBAB and [bmim] $\mathrm{PF}_{6}$, catalyzed by palladium acetate with and without phosphorus ligands. Compared to the conventional solvent media which are used for Heck couplings, ILs are not only environmentally friendly and facilitate catalyst and reaction product recovery, they are highly stereoselective as well.

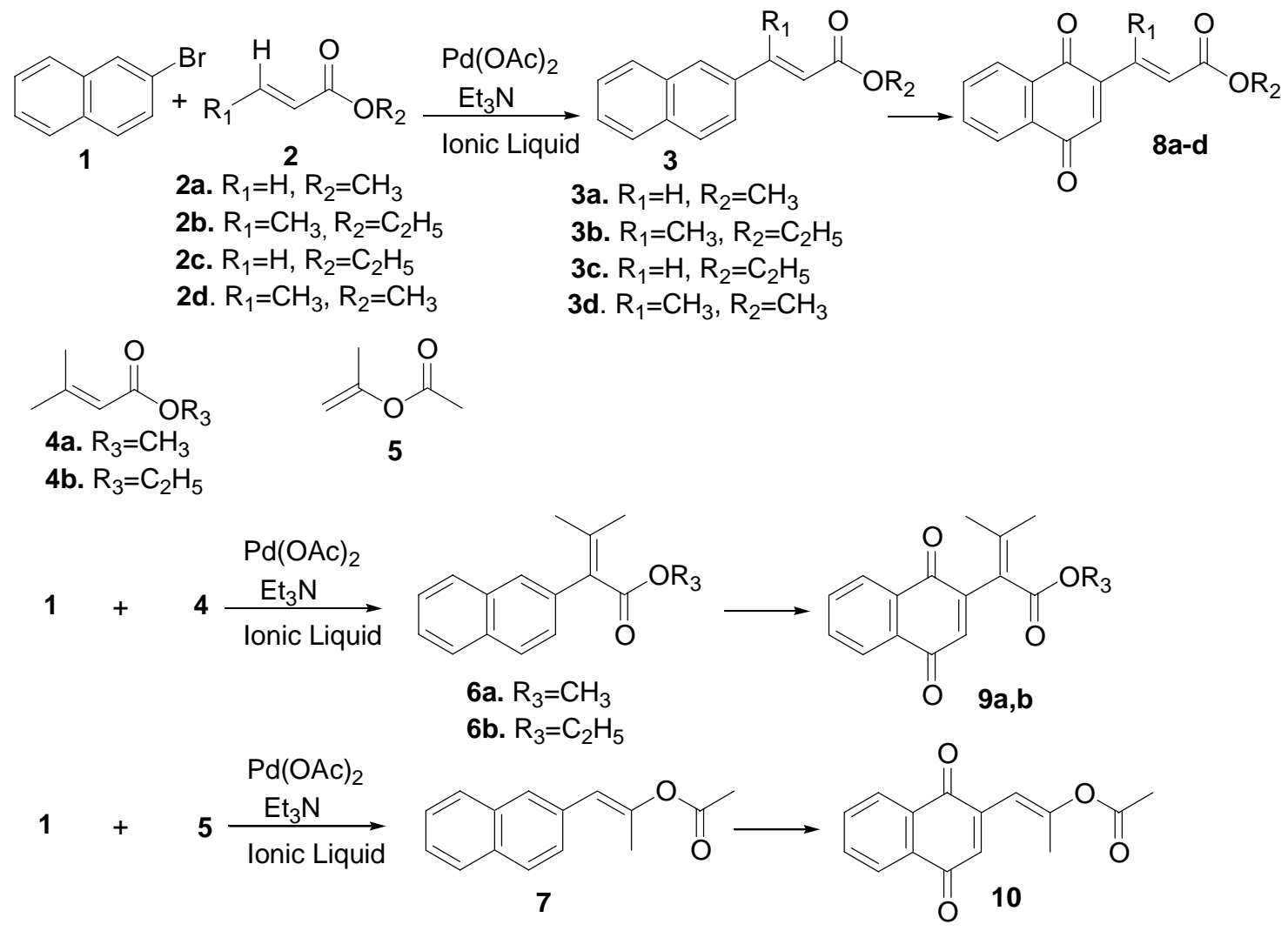

Scheme 1. Heck coupling of 2-bromonaphthalene with various vinyl esters and oxidation of reaction products 
Table 1. Heck coupling of 2-bromonaphthalene 1 with various vinyl esters ${ }^{\mathrm{a}}$

\begin{tabular}{|c|c|c|c|c|c|}
\hline S. No & Substrate & Ionic liquid & Temp. $\left({ }^{\circ} \mathrm{C}\right)$ & Time (hr) & $\begin{array}{c}\text { \%Yield }{ }^{\mathrm{C}} \\
\text { (Product no) }\end{array}$ \\
\hline 1 & & TBAB & 120 & 4 & $80(3 a)$ \\
\hline & $2 \mathrm{a}$ & & & & \\
\hline 2 & $2 \mathrm{a}$ & {$[\mathrm{bmim}] \mathrm{PF}_{6}$} & 70 & 4 & 98 (3a) \\
\hline 3 & $2 \mathrm{a}$ & {$[\mathrm{bmim}] \mathrm{PF}_{6}$} & 100 & 4 & $98(3 a)$ \\
\hline 4 & $2 a$ & $\begin{array}{c}{\text { [bmim] } \mathrm{PF}_{6}} \\
+\mathrm{TBAB}^{\mathrm{b}}\end{array}$ & 70 & 3 & 98 (3a) \\
\hline 5 & $2 b$ & TBAB & 120 & 4 & 70 (3b) \\
\hline 6 & $2 b$ & [bmim] $\mathrm{PF}_{6}$ & 70 & 4 & 80 (3b) \\
\hline 7 & $2 b$ & $\begin{array}{c}{[\mathrm{bmim}] \mathrm{PF}_{6}} \\
+\mathrm{TBAB}^{\mathrm{b}}\end{array}$ & 70 & 3 & $82(3 b)$ \\
\hline 8 & 2c & {$[\mathrm{bmim}] \mathrm{PF}_{6}$} & 70 & 3 & $80(3 c)$ \\
\hline 9 & $2 c$ & $\begin{array}{c}{[\mathrm{bmim}] \mathrm{PF}_{6}} \\
+\mathrm{TBAB}^{\mathrm{b}}\end{array}$ & 70 & 3 & 81 (3c) \\
\hline 10 & $2 d$ & {$[\mathrm{bmim}] \mathrm{PF}_{6}$} & 70 & 3 & 80 (3d) \\
\hline 11 & $2 d$ & $\begin{array}{c}\text { [bmim] }^{\mathrm{bF}_{6}} \\
+\mathrm{TBAB}^{\mathrm{b}}\end{array}$ & 70 & 3 & 82 (3d) \\
\hline 12 & & TBAB & 120 & 4 & $60(6 a)$ \\
\hline & $4 a$ & & & & \\
\hline 13 & $4 a$ & {$[\mathrm{bmim}] \mathrm{PF}_{6}$} & 70 & 4 & $70(6 a)$ \\
\hline 14 & $4 a$ & $\begin{array}{c}{\left[\mathrm{bmim}^{2} \mathrm{PF}_{6}\right.} \\
+\mathrm{TBAB}^{\mathrm{b}}\end{array}$ & 70 & 3 & $72(6 a)$ \\
\hline 15 & $4 \mathrm{~b}$ & {$[\mathrm{bmim}] \mathrm{PF}_{6}$} & 70 & 4 & $72(6 b)$ \\
\hline 16 & $4 \mathrm{~b}$ & $\begin{array}{c}{[\mathrm{bmim}] \mathrm{PF}_{6}} \\
+\mathrm{TBAB}^{\mathrm{b}} \\
\end{array}$ & 70 & 3 & 75 (6b) \\
\hline 17 & & TBAB & 120 & 4 & $55(7)$ \\
\hline & 5 & & & & \\
\hline 18 & 5 & {$[\mathrm{bmim}] \mathrm{PF}_{6}$} & 70 & 4 & $78(7)$ \\
\hline 19 & 5 & $\begin{array}{c}{[\mathrm{bmim}] \mathrm{PF}_{6}} \\
+\mathrm{TBAB}^{\mathrm{b}}\end{array}$ & 70 & 3 & $86(7)$ \\
\hline
\end{tabular}

${ }^{a}$ Reactions carried out using palladium acetate as the catalyst, triethylamine as base and tri-otolylphosphine as a ligand; ${ }^{\mathrm{b}}$ Catalytic amount of TBAB used without any ligand; ${ }^{\mathrm{c}}$ Isolated yield, average of three experiments. 


\section{Experimental Section}

General Procedures. All reagents and chemicals were obtained from Aldrich Chemical Company (USA) and were used as received unless otherwise noted. Solvents used for the chemical synthesis were of laboratory and analytical grade, and were used without further purification. Thin layer chromatography analyses were performed on precoated silica gel plates (Merck F254, $0.2 \mathrm{~mm}$ thickness). Column chromatography was performed on flash silica gel (230-400 mesh). Melting points were recorded on a Buchi capillary melting point apparatus and are uncorrected. Infrared (IR) spectra were recorded on a Nicolet Impact-410 FTIR spectrometer. Proton magnetic resonance $\left({ }^{1} \mathrm{H}\right.$ NMR) spectra were recorded on a Bruker $300 \mathrm{MHz}$ spectrometer in $\mathrm{CDCl}_{3}$ or $\mathrm{DMSO}-\mathrm{d}_{6}$ solution. The chemical shifts are reported in $\delta(\mathrm{ppm})$ relative to internal standard tetramethylsilane (TMS) and coupling constants J are given in Hz. Mass spectrometry was conducted using GCMS ( Shimadzu QP 5000 mass spectrometer) auto sampler/direct injection (EI/CI). C, H elemental analysis was done on Elementar Vario EL analyzer.

\section{General procedure for the Heck reactions in ionic liquid}

3-Naphthalen-2-yl-acrylic acid methyl ester (3a). The ionic liquid [bmim] $\mathrm{PF}_{6}(1.4 \mathrm{ml})$, palladium acetate $(0.23 \mathrm{~g}, 0.12 \mathrm{mmol})$, and tris-o-tolylphosphine $(0.73 \mathrm{~g}, 0.24 \mathrm{mmol})$ were mixed in a three-necked round bottom flask and heated to $120^{\circ} \mathrm{C}$ under nitrogen atmosphere for 10-15 minutes till a yellowish brown solution of catalyst in the ionic liquid was obtained. Methyl acrylate (2a, $0.29 \mathrm{~g}, 2.90 \mathrm{mmol})$, triethylamine $(0.37 \mathrm{~g}, 3.63 \mathrm{mmol})$ and 2-bromonaphthalene (1, $0.5 \mathrm{~g}, 2.42 \mathrm{mmol}$ ) were added to the solution and the reaction mixture has been heated at $70{ }^{\circ} \mathrm{C}$ for 4 hours. After the completion of the reaction (monitored by TLC), n-hexane (10ml) was added to the reaction mixture and extraction was done for 15 minutes with vigorous stirring. The extraction was repeated two times. The organic extracts were combined and concentrated under reduced pressure to give the crude product. Recrystallization of the crude product from hexane afforded $0.502 \mathrm{~g}$ of 3 a as a white crystalline solid in $98 \%$ yield. M.p. $96-98{ }^{\circ} \mathrm{C}$; IR $\left(\mathrm{KBr}, \mathrm{cm}^{-1}\right)$ : 3055, 2925, 1730, 1714, 1646, 1436, 1313, 1273, 1163, 824; GCMS m/z: $212\left(\mathrm{M}^{+}\right) ;{ }^{1} \mathrm{H}$ NMR $\left(\mathrm{CDCl}_{3}\right): \delta 7.93$ (s, $\left.1 \mathrm{H}\right), 7.85-7.83(\mathrm{~m}, 4 \mathrm{H}), 7.67(\mathrm{~d}, \mathrm{~J}=8.4 \mathrm{~Hz}, 1 \mathrm{H}), 7.52-7.49(\mathrm{~m}, 2 \mathrm{H}), 6.56(\mathrm{~d}$, $J=15.8 \mathrm{~Hz}, 1 \mathrm{H}$ ), 3.83 (s, 3H); Anal. Calc for $\mathrm{C}_{14} \mathrm{H}_{12} \mathrm{O}_{2}$ : C, 79.25 \%; H, 5.66 \%. Found: C, 79.40 \%; H, $5.60 \%$.

3-Naphthalen-2-yl-but-2-enoic acid ethyl ester (3b). From the reaction with ethyl-transcrotonate $\mathbf{2} \mathbf{b}, \mathbf{3} \mathbf{b}$ was obtained as a greenish white crystalline solid in $80 \%$ yield. The crude product was purified by silica gel column chromatography (Hexane: Ethyl acetate, 98:2). M.p. 44-45 ${ }^{\circ} \mathrm{C}$; IR $\left(\mathrm{KBr}, \mathrm{cm}^{-1}\right)$ : 3057, 2854, 1729, 1714, 1629, 1504, 1435, 1315, 1195, 748; GCMS m/z: $240\left(\mathrm{M}^{+}\right) ;{ }^{1} \mathrm{H}$ NMR $\left(\mathrm{CDCl}_{3}\right): \delta 7.94(\mathrm{~s}, 1 \mathrm{H}), 7.83-7.80(\mathrm{~m}, 3 \mathrm{H}), 7.60(\mathrm{~d}, \mathrm{~J}=8.4 \mathrm{~Hz}, 1 \mathrm{H})$, 7.54-7.49 (m, 2H), 6.28 (s, 1H), 4.20 (q, 2H), 2.68 (s, 3H), 1.35 (s, 3H); Anal. Calc for $\mathrm{C}_{16} \mathrm{H}_{16} \mathrm{O}_{2}$ : C, $80.0 \%$; H, $6.66 \%$. Found: C, $80.0 \%$; H, $6.65 \%$.

3-naphthalen-2-yl-acrylic acid ethyl ester (3c). From the reaction with ethyl acrylate 2c, 3c was obtained as a crystalline solid in $80 \%$ yield by purification of the crude product as in example 2. M.p. $95^{\circ} \mathrm{C}$; IR (KBr, $\left.\mathrm{cm}^{-1}\right)$ : 3055, 1730, 1715, 1645, 1435, 1313, 1273, 1192, 983, 
825; GCMS m/z: $226\left(\mathrm{M}^{+}\right)$; ${ }^{1} \mathrm{H}$ NMR $\left(\mathrm{CDCl}_{3}\right): \delta 7.93$ (s, 1H), 7.85-7.83 (m, 4H), 7.67 (d, J=8.4 $\mathrm{Hz}, 1 \mathrm{H}), 7.54-7.49$ (m, 2H), 6.28 (s, $1 \mathrm{H}), 4.20$ (q, 2H), 1.35 (s, 3H); Anal. Calc for $\mathrm{C}_{15} \mathrm{H}_{14} \mathrm{O}_{2}$ : C, 79.65 \%; H, 6.19 \%. Found: C, $79.55 \%$; H, $6.15 \%$.

3-naphthalen-2-yl-but-2-enoic acid methyl ester (3d). From the reaction with methyl-transcrotonate 2d, 3d was obtained as a white crystalline solid in $80 \%$ yield by purification of the crude product as in example 2. M.p. 50-52 ${ }^{\circ} \mathrm{C}$; IR $\left(\mathrm{KBr}, \mathrm{cm}^{-1}\right)$ : 3057, 2854, 1730, 1714, 1629, 1504, 1435, 1320, 1195, 950, 822, 748; GCMS m/z: $226\left(\mathrm{M}^{+}\right) ;{ }^{1} \mathrm{H}$ NMR $\left(\mathrm{CDCl}_{3}\right): \delta 7.94(\mathrm{~s}, 1 \mathrm{H})$, 7.83-7.80 (m, 3H), 7.60 (d, J=8.4 Hz, 1H), 7.54-7.49 (m, 2H), 6.28 (s, 1H), 3.85 (s, 3H), 1.85 (s, $3 \mathrm{H}$ ); Anal. Calc for $\mathrm{C}_{15} \mathrm{H}_{14} \mathrm{O}_{2}$ : C, $79.65 \%$; H, $6.19 \%$. Found: C, 79.50 \%; H, $6.15 \%$.

3-methyl-2-naphthalen-2-yl-but-2-enoic acid methyl ester (6a). From the reaction with methyl-3,3-dimethyl acrylate 4a, 6a was obtained as a brown crystalline solid in $70 \%$ yield by purification of the crude product as in example 2. M.p. $120-122{ }^{\circ} \mathrm{C}$; IR $\left(\mathrm{KBr}, \mathrm{cm}^{-1}\right)$ : 3055, 2925, 1736, 1728, 1645, 1591, 1548, 1436, 1377, 1160, 956, 790; GCMS m/z: $240\left(\mathrm{M}^{+}\right)$; ${ }^{1} \mathrm{H}$ NMR $\left(\mathrm{CDCl}_{3}\right): \delta 8.0(\mathrm{~d}, 1 \mathrm{H}), 7.84-7.76(\mathrm{~m}, 3 \mathrm{H}), 7.60-7.48(\mathrm{~m}, 3 \mathrm{H}), 3.67(\mathrm{~s}, 3 \mathrm{H}), 1.88(\mathrm{~s}, 6 \mathrm{H})$; Anal. Calc for $\mathrm{C}_{16} \mathrm{H}_{16} \mathrm{O}_{2}$ : C, 80.0 \%; H, 6.66 \%. Found: C, $80.0 \%$; H, 6.60 \%.

3-methyl-2-naphthalen-2-yl-but-2-enoic acid ethyl ester (6b). From the reaction with ethyl3,3-dimethyl acrylate $\mathbf{4 b}, \mathbf{6 b}$ was obtained as a brown crystalline solid in $72 \%$ yield by purification of the crude product as in example 2. M.p. $102-103{ }^{\circ} \mathrm{C}$; IR $\left(\mathrm{KBr}, \mathrm{cm}^{-1}\right)$ : 3055, 2925, 1736, 1728, 1645, 1591, 1548, 1436, 1377, 1160, 956, 790; GCMS m/z: $254\left(\mathrm{M}^{+}\right)$; ${ }^{1} \mathrm{H}$ NMR $\left(\mathrm{CDCl}_{3}\right): \delta 8.0(\mathrm{~d}, 1 \mathrm{H}), 7.84-7.76(\mathrm{~m}, 3 \mathrm{H}), 7.60-7.48(\mathrm{~m}, 3 \mathrm{H}), 4.20(\mathrm{q}, 2 \mathrm{H}), 1.88(\mathrm{~s}, 6 \mathrm{H}), 1.35(\mathrm{~s}$, $3 \mathrm{H}$ ); Anal. Calc for $\mathrm{C}_{17} \mathrm{H}_{18} \mathrm{O}_{2}$ : C, $80.32 \%$; 7.09 \%. Found: C, $80.0 \%$; $7.00 \%$.

Acetic acid 1-methyl-2-naphthalen-2-yl-vinylester (7). From the reaction with isopropenyl acetate 5, 7 was obtained as a brown crystalline solid in $78 \%$ yield by purification of the crude product as in example 2. M.p. 52-54 ${ }^{\circ} \mathrm{C}$; IR $\left(\mathrm{KBr}, \mathrm{cm}^{-1}\right)$ : 3054, 2852, 1754, 1631, 1598, 1455 , 1368, 1217, 1130, 816, 746; GCMS m/z: $226\left(\mathrm{M}^{+}\right) ;{ }^{1} \mathrm{H}$ NMR $\left(\mathrm{CDCl}_{3}\right): \delta 8.00(\mathrm{~s}, 1 \mathrm{H}), 7.83-7.70$ (m, 3H), 7.56-7.46 (m, 3H), $6.51(\mathrm{~s}, 1 \mathrm{H}), 2.37$ (s, 3H) 1.96 (s, 3H); Anal. Calc for $\mathrm{C}_{15} \mathrm{H}_{14} \mathrm{O}_{2}$ : C, $79.65 \%$; H, 6.19 \%. Found: C, $79.60 \%$; H, $6.20 \%$.

\section{General procedure for the oxidation of 2-substituted naphthalene derivatives}

To an ice-cooled solution of periodic acid $(4.2 \mathrm{~mol})$ and chromium trioxide $(0.1 \mathrm{~mol})$ in acetonitrile was added 2-substituted naphthalene derivative $(1 \mathrm{~mol})$ all at once. A white precipitate was formed immediately with exothermic reaction. After stirring the reaction at $5{ }^{\circ} \mathrm{C}$ for an hour, the supernatant liquid of the reaction mixture was removed and concentrated. The residues after decantation and evaporation were dissolved in water and methylene chloride, combined and then extracted with methylene chloride. The organic extracts were washed with aqueous sodium hydroxide solution, brine and then dried over anhydrous sodium sulphate. The solvent was distilled off under reduced pressure. The product was purified and separated by silica gel column chromatography.

3-(1,4-dioxo-1,4-dihydro-naphthalen-2-yl)-acrylic acid methyl ester (8a). From the reaction with 3-naphthalen-2-yl-acrylic acid methyl ester 3a, 8a was obtained as viscous oil in 61\% yield. IR (KBr, $\left.\mathrm{cm}^{-1}\right)$ : 3058, 1728, 1714, 1665, 1642, 1594, 1441, 1314, 1272, 1172, 983, 824; GCMS 
m/z: $242\left(\mathrm{M}^{+}\right) ;{ }^{1} \mathrm{H}$ NMR $\left(\mathrm{CDCl}_{3}\right): \delta$ 7.99-7.92 (m, 2H), 7.86-7.80 (m, 3H), 7.52-7.49 (m, $\left.1 \mathrm{H}\right)$, $6.28(\mathrm{~d}, J=15.8 \mathrm{~Hz}, 1 \mathrm{H}), 3.83$ (s, 3H); Anal. Calc for $\mathrm{C}_{14} \mathrm{H}_{10} \mathrm{O}_{4}$ : C, $69.42 \%$; H, $4.16 \%$. Found: C, $69.60 \%$; H, $4.20 \%$.

3-(1,4-dioxo-1,4-dihydro-naphthalen-2-yl)-but-2-enoic acid ethyl ester (8b). From the reaction with 3-naphthalen-2-yl-but-2-enoic acid ethyl ester $\mathbf{3 b}$, $\mathbf{8 b}$ was obtained as a viscous liquid in $50 \%$ yield. IR $\left(\mathrm{KBr}, \mathrm{cm}^{-1}\right)$ : 3057, 2854, 1730, 1714, 1655, 1629, 1504, 1195, 748; GCMS m/z: $270\left(\mathrm{M}^{+}\right) ;{ }^{1} \mathrm{H}$ NMR $\left(\mathrm{CDCl}_{3}\right): \delta$ 7.98-7.90 (m, 2H), 7.83-7.80 (m, 3H), $6.28(\mathrm{~s}, 1 \mathrm{H})$, 4.20 (q, 2H), 1.80 (s, 3H), 1.35 (s, 3H); Anal. Calc for $\mathrm{C}_{16} \mathrm{H}_{14} \mathrm{O}_{4}$ : C, $71.10 \%$; H, $5.22 \%$. Found: C, $71.50 \%$; $\mathrm{H}, 5.50 \%$.

3-(1,4-dioxo-1,4-dihydro-naphthalen-2-yl)-acrylic acid ethyl ester (8c). From the reaction with 3-naphthalen-2-yl-acrylic acid ethyl ester 3c, 8c was obtained as a viscous liquid in $52 \%$ yield. IR (KBr, $\left.\mathrm{cm}^{-1}\right)$ : 3055, 1730, 1715, 1660, 1645, 1435, 1313, 1273, 1192, 983, 825; GCMS m/z: $256\left(\mathrm{M}^{+}\right) ;{ }^{1} \mathrm{H}$ NMR $\left(\mathrm{CDCl}_{3}\right): \delta$ 7.98-7.94 (m, 2H), 7.85-7.83 (m, 3H), 7.20 (d, J=8.4 Hz, $1 \mathrm{H}), 6.28(\mathrm{~s}, 1 \mathrm{H}), 4.20$ (q, 2H), 1.35 (s, 3H); Anal. Calc for $\mathrm{C}_{15} \mathrm{H}_{12} \mathrm{O}_{4}$ : C, $70.31 \%$; $4.72 \%$. Found: C, $70.60 \%$; H, $4.75 \%$.

3-(1,4-dioxo-1,4-dihydro-naphthalen-2-yl)-but-2-enoic acid methyl ester (8d). From the reaction with 3-naphthalen-2-yl-but-2-enoic acid methyl ester 3d, 8d was obtained as a viscous liquid in 52\% yield. IR $\left(\mathrm{KBr}, \mathrm{cm}^{-1}\right)$ : 3057, 2854, 1730, 1714, 1662, 1629, 1504, 1195, 982, 748; GCMS m/z: $256\left(\mathrm{M}^{+}\right) ;{ }^{1} \mathrm{H}$ NMR $\left(\mathrm{CDCl}_{3}\right): \delta$ 7.98-7.94 (m, 2H), 7.83-7.80 (m, 3H), $6.28(\mathrm{~s}, 1 \mathrm{H})$, 3.85 (s, 3H), 1.85 (s, 3H); Anal. Calc for $\mathrm{C}_{15} \mathrm{H}_{12} \mathrm{O}_{4}$ : C, $70.31 \%$; $\mathrm{H}, 4.72 \%$. Found: C, $70.60 \%$; $\mathrm{H}, 4.75 \%$.

2-(1,4-dioxo-1,4-dihydro-naphthalen-2-yl)-3-methyl-but-2-enoic acid methyl ester (9a). From the reaction with 3-methyl-2-naphthalen-2-yl-but-2-enoic acid methyl ester 6a, 9a was obtained as a viscous liquid in 55\% yield. IR $\left(\mathrm{KBr}, \mathrm{cm}^{-1}\right)$ : 3055, 2925, 1736, 1728, 1645, 1591 , 1548, 1436, 1377, 1160, 956, 790; GCMS m/z: $270\left(\mathrm{M}^{+}\right) ;{ }^{1} \mathrm{H}$ NMR $\left(\mathrm{CDCl}_{3}\right): \delta 8.0-7.98$ (m, 2H), 7.84-7.76 (m, 3H), 3.67 (s, 3H), 1.88 (s, 6H); Anal. Calc for $\mathrm{C}_{16} \mathrm{H}_{14} \mathrm{O}_{4}: \mathrm{C}, 71.10 \%$; $5.22 \%$. Found: C, $71.55 \%$; H, $5.50 \%$.

2-(1,4-dioxo-1,4-dihydro-naphthalen-2-yl)-3-methyl-but-2-enoic acid ethyl ester (9b). From the reaction with 3-methyl-2-naphthalen-2-yl-but-2-enoic acid ethyl ester $\mathbf{6 b}, \mathbf{9 b}$ was obtained as a viscous liquid in $60 \%$ yield. IR $\left(\mathrm{KBr}, \mathrm{cm}^{-1}\right)$ : 3055, 2925, 1736, 1728, 1665, 1645, 1590, 1546, 1432, 1378, 1160, 956, 790; GCMS m/z: $284\left(\mathrm{M}^{+}\right) ;{ }^{1} \mathrm{H}$ NMR $\left(\mathrm{CDCl}_{3}\right): \delta 8.0-7.98$ (m, 2H), 7.847.76 (m, 3H), 4.20 (q, 2H), 1.86 (s, 6H), 1.35 (s, 3H); Anal. Calc for $\mathrm{C}_{17} \mathrm{H}_{16} \mathrm{O}_{4}$ : C, $71.82 \%$; $\mathrm{H}$, $5.67 \%$. Found: C, $72.00 \%$; H, $5.80 \%$.

Acetic acid-2-(1,4-dioxo-1,4-dihydro-naphthalen-2-yl)-1-methyl-vinyl ester (10). From the reaction with acetic acid 1-methyl-2-naphthalen-2-yl-vinylester 7, 10 was obtained as a viscous liquid in 54\% yield. IR $\left(\mathrm{KBr}, \mathrm{cm}^{-1}\right)$ : 3054, 2852, 1754, 1715, 1662, 1631, 1598, 1455, 1368, 1217, 1130, 816, 746; GCMS m/z: $256\left(\mathrm{M}^{+}\right) ;{ }^{1} \mathrm{H}$ NMR $\left(\mathrm{CDCl}_{3}\right): \delta$ 8.00-7.98 (m, 2H), 7.86-7.78 (m, 3H), 6.46 (s, 1H), 2.35 (s, 3H) 1.96 (s, 3H); Anal. Calc for $\mathrm{C}_{15} \mathrm{H}_{12} \mathrm{O}_{4}$ : C, $70.31 \%$; H, $4.72 \%$. Found: C, $70.50 \%$; H, $4.75 \%$. 


\section{References}

1. Tran, T.; Saheba, E.; Arcerio, A. V.; Chavez, V.; Li, Q-yi.; Martinez, L. E.; Primm, T. P. Bioorg. Med. Chem. 2004, 12, 4809.

2. Huang, S.-T.; Kuo, H.-S.; Hsiao, C.-L.; Lin, Y.-L. Bioorg. Med. Chem. 2002, 10, 1947.

3. Tandon, V. K.; Chhor, R. B.; Singh, R. V.; Rai, S.; Yadav, D. B. Bioorg. Med. Chem. Lett. 2004, 14, 1079.

4. DaSilva, A. J. M.; Buarque, C. D.; Brito, F. V.; Aurelian, L.; Macedo, L. F.; Malkas, L. H.; Hickey, R. J.; Lopes, D. V. S.; Noel, F.; Murakami, Y. L. B.; Silva, N. M. V.; Melo, P. A.; Caruso, R. R. B.; Castro, N. G.; Costa, P. R. R. Bioorg. Med. Chem. 2002, 10, 2731.

5. Calo, V.; Nacci, A.; Monopoli, A. J. Mol. Catalysis A: Chemical. 2004, 214, 45.

6. Yamazaki, S. Tetrahedron Lett. 2001, 42, 3355.

7. Wagner, M. Chimica Oggi. 2004, 6, 17.

8. McNulty, J.; Capretta, A.; Cheekoori, S.; Clyburne, J. A. C.; Robertson, A. J. Chimica Oggi. 2004, 22, 13.

9. Trzeciak, A. M.; Ziolkowski, J. J. Coord. Chem. Rev. 2005, 249, 2308.

10. Carmichael, A. J.; Earle, E. J.; Holbrey, J. D.; Mccormac, P. B.; Seddon, K. R. Org. Lett. 1999, 1, 997.

11. Zou, G.; Wang, Z.; Zhu, J.; Tang, J.; He, M. Y. J. Mol. Catalysis A: Chemical 2003, 206, 193.

12. Schmidt, A. F.; Smirnov, V. V. J. Mol. Catalysis A: Chemical 2003, 203, 75.

13. Earle, M. J.; Seddon, K. R. Pure Appl. Chem. 2000, 72, 1391.

14. Cavell, K. J.; McGuiness, D. S. Coord. Chem. Rev. 2004, 248, 671. 